\title{
Connectivity of Connected Bipartite Graphs with Two Orbits
}

\author{
Xiaodong Liang and Jixiang Meng* \\ College of Mathematics and System Sciences,Xinjiang University \\ Urumqi,Xinjiang 830046, People's Republic of China \\ liangxd-1970@163.com, \\ mjx@xju.edu.cn
}

\begin{abstract}
Let $X=(V, E)$ be a simple connected graph, the connectivity $\kappa(X)$ of $X$ is the minimum cardinality of all vertex cut sets in $X$. Let $x \in V(X)$, the set $\left\{x^{g}: g \in \operatorname{Aut}(X)\right\}$ is called an orbit of $\operatorname{Aut}(X)$, the automorphism group of $X$. In this note, we prove that the connectivity of a connected bipartite graph with two orbits attains its minimum degree.
\end{abstract}

Keywords: Connectivity, Bipartite graph, Orbit.

\section{Introduction}

Let $X=(V, E)$ be a simple connected graph, with $V(X)$ the set of vertices and $E(X)$ the set of edges. A vertex cut of $X$ is a subset $U$ of $V$ such that the subgraph $X \backslash U$ induced by $V \backslash U$ is either trivial or not connected. The connectivity $\kappa(X)$ of a nontrivial connected graph $X$ is the minimum cardinality of all vertex cut sets of $X$. If we denote by $\delta(X)$ the minimum degree of $X$, then $\kappa(X) \leq \delta(X)$.

We denote by $A u t(X)$ the automorphism group of $X$. The graph $X$ is said to be vertex transitive if for any two distinct vertices of $X$ there is an automorphism mapping one to the other. Let $x \in V(X)$, we call the set $\left\{x^{g}: g \in \operatorname{Aut}(X)\right\}$ an orbit of $A u t(X)$. Clearly, $A u t(X)$ acts transitively on each orbit of $A u t(X)$.

In [3], Mader proved that the connectivity of a connected vertex transitive graph, which is $K_{4}-$ free, attains its minimum degree. It arouses us to consider the relation between the connectivity and the number of orbits. In this note, we prove that the connectivity of a connected bipartite graph with two orbits attains its minimum degree.

In the next, we introduce some terms and theorems which will be used in proving our main results.

Let $X=(V, E)$ be a connected graph and $F$ be a non-empty subset of $V(G)$. Set $N(F)=\{x \in V(X) \backslash F: \exists y \in F$, st. $\quad x y \in E(X)\}, C(F)=F \cup N(F)$ and $R(F)=V(X) \backslash C(F)$. Clearly, $N(F)$ is a vertex cut set if $R(F) \neq \emptyset$. A subset $F \subset V(X)$ is said to be a fragment if $|N(F)|=\kappa(X)$ and $R(F) \neq \emptyset$. A fragment of minimum cardinality is called an atom of $X$. An imprimitive block for a group

* Correspondence author, the research of this author is supported by NSFC, FUKT and SPUX. 
$U$ of permutations on a set $T$ is a proper, nontrivial subset $A$ of $T$ such that if $\sigma \in U$ then either $\sigma(A)=A$ or $\sigma(A) \cap A=\emptyset$. A subset $A$ of $V(X)$ is called an imprimitive block for $X$ if it is an imprimitive block for $A u t(X)$ on $V(X)$.

Theorem 1. [8] Let $X=(V, E)$ be a nontrivial connected graph which is not a complete graph.

(i) $\kappa(X)=\delta(X)$ if and only if every atom of $X$ has cardinality 1;

(ii) if $\kappa(X)<\delta(X)$, then each atom has cardinality at most $[(|V|-\kappa(X)) / 2]$ and induces a connected subgraph of $X$.

Theorem 2. 5] If $X=(V, E)$ is a nontrivial connected graph which is not a complete graph, then distinct atoms of $X$ are disjoint. Thus if $\kappa(X)<\delta(X)$, the atoms of $X$ are imprimitive blocks of $X$.

Theorem 3. [5] Let $X=(V, E)$ be a nontrivial connected graph. If $W$ is a minimum vertex cut set and $A$ an atom of $X$, then $A \cap W=\emptyset$ or $A \subseteq W$.

\section{Main Results}

Let $X=(V, E)$ be a connected bipartite graph with two orbits. Clearly, it is semiregular. In this section, we use $X_{0}$ and $X_{1}$ to denote the two orbits of $\operatorname{Aut}(X)$. Without loss of generality, we may assume that $m=d(u)$ for any $u \in X_{0}$, $n=d(v)$ for any $v \in X_{1}$, and $m \leq n$. So we have $\delta(X)=m$. Let $A$ be an atom of $X$. Set $A_{0}=A \cap X_{0}$ and $A_{1}=A \cap X_{1}$, then $A=A_{0} \cup A_{1}$.

Lemma 1. Let $X=(V, E)$ be a connected bipartite graph with two orbits, and $A$ be an atom of $X$. If $\kappa(X)<\delta(X)$, then $A_{i}=A \cap X_{i}(i=0,1)$ are nontrivial.

Proof. By Theorem [1] the induced subgraph $Y=X[A]$ is a nontrivial connected subgraph of $X$, which is a bipartite graph, thus $A_{i}=A \cap X_{i} \neq \emptyset(i=0,1)$. Suppose that one of these two vertex subsets is trivial.

Case 1: $\left|A_{0}\right|=1$. Thus, $\left|A_{1}\right| \leq m$ since $Y$ is connected. Set $F=N(A)$.

Subcase 1.1: $\left|A_{1}\right|=m$. So we have $|F| \geq n-1$. If $|F|=n-1$, then the induced subgraph $Y^{\prime}=X[A \cup F]$ is a connected component of $X$, which is impossible since $X$ is connected. Thus $|F|>n-1$, namely $|F| \geq n \geq m=\delta(X)$, a contradiction.

Subcase 1.2: $\left|A_{1}\right|=p \leq m-1$. Thus $\left|N\left(A_{0}\right) \backslash A_{1}\right|=m-p$. Let $q=\left|N\left(A_{1}\right) \backslash A_{0}\right|$, we have $q \geq n-1$. Since

$$
|F|=|N(A)|=\left|N\left(A_{0}\right) \backslash A_{1}\right|+\left|N\left(A_{1}\right) \backslash A_{0}\right|=m-p+q \geq m+n-p-1
$$

and $|F|=\kappa(X)<m$, we have $n<p+1 \leq m$, a contradiction.

Case 2: $\left|A_{1}\right|=1$. Thus, $\left|A_{0}\right| \leq n$ since $Y$ is connected. Set $F=N(A)$. 
Subcase 2.1: $\left|A_{0}\right|=n$. So we have $|F| \geq m-1$. Since $|F|=\kappa(X)<m,|F| \leq m-1$. Namely, $|F|=m-1$. Thus we have that the induced subgraph $Y^{\prime}=X[A \cup F]$ is a connected component of $X$, a contradiction.

Subcase 1.2: $\left|A_{0}\right|=p \leq n-1$. Thus $\left|N\left(A_{0}\right) \backslash A_{1}\right|=n-p$. Let $q=\left|N\left(A_{1}\right) \backslash A_{0}\right|$, then $q \geq m-1$. Since

$$
|F|=|N(A)|=\left|N\left(A_{0}\right) \backslash A_{1}\right|+\left|N\left(A_{1}\right) \backslash A_{0}\right|=n-p+q \geq n+m-p-1
$$

and $|F|=\kappa(X)<m$, we have $n<p+1 \leq n$, a contradiction.

The result follows.

Lemma 2. Let $X=(V, E)$ be a connected bipartite graph with two orbits, and $A$ be an atom of $X$, and $Y=X[A]$. If $\kappa(X)<\delta(X)$, then Aut $(Y)$ acts transitively on $A_{i}=A \cap X_{i}(i=0,1)$.

Proof. By Lemma 1, $A_{i}=A \cap X_{i}(i=0,1)$ are nontrivial. For any $u, v \in A_{0}$, there exists an automorphism $\varphi$ of $X$ with $\varphi(u)=v$ and so $\varphi(A) \cap A \neq \emptyset$. By Theorem 2. $\varphi(A)=A$. Since $A_{0}$ and $A_{1}$ are contained in different orbits, we have $\varphi\left(A_{0}\right)=A_{0}$ and $\varphi\left(A_{1}\right)=A_{1}$. Thus the restriction of $\varphi$ on $A$ induces an automorphism of $Y$, and then $A u t(Y)$ acts transitively on $A_{0}$. Similarly, Aut $(Y)$ acts transitively on $A_{1}$. The result follows.

Lemma 3. Let $X=(V, E)$ be a connected bipartite graph with two orbits, and $A$ be an atom of $X$. If $\kappa(X)<\delta(X)$, then

(i) Every vertex of $X$ lies in an atom;

(ii) $|A| \leq \kappa(X)$.

Proof. (i) By Lemma 1, the induced subgraph $Y=X[A]$ is a nontrivial connected subgraph of $X$, thus at least one vertex of $X_{i}(i=0,1)$, respectively, lies in an atom. By the transitivity of $X_{i}$, every vertex of $X$ lies in an atom.

(ii) Let $F=N(A)$. By (i), for any $u \in F, u$ lies in an atom $A^{\prime}$ of $X$. By Theorem 3. $A^{\prime} \subseteq F$, then $|A|=\left|A^{\prime}\right| \leq|F|=\kappa(X)$.

Theorem 4. If $X=(V, E)$ is a connected bipartite graph with two orbits, then $\kappa(X)=\delta(X)$.

Proof. Suppose to the contrary that $\kappa(X)<\delta(X)$. By Theorem 1 and Lemma 3 , $V(X)$ is a disjoint union of distinct atoms. Let $A$ be an atom of $X$, then there exist $\sigma_{i} \in \operatorname{Aut}(X)(i=1, \cdots, k)$, such that

$$
V(X)=\bigcup_{i=1}^{k} \sigma_{i}(A) .
$$

By Lemma 1, the induced subgraph $Y=X[A]$ is a nontrivial connected subgraph of $X$, and $A_{i}=A \cap X_{i}(i=0,1)$ are nontrivial. Since $\operatorname{Aut}(X)$ has two orbits $X_{0}, X_{1}$, for any $1 \leq i, j \leq k$ and $i \neq j, \sigma_{i}\left(A_{0}\right) \cap \sigma_{j}\left(A_{0}\right)=\emptyset$ and $\sigma_{i}\left(A_{0}\right), \sigma_{j}\left(A_{0}\right) \subseteq X_{0}$. 
Thus, we have $X_{0}=\bigcup_{i=1}^{k} \sigma_{i}\left(A_{0}\right), X_{1}=\bigcup_{i=1}^{k} \sigma_{i}\left(A_{1}\right)$, and $\left|A_{i}\right||| X_{i} \mid(i=0,1)$. Since $\left|X_{0}\right| /\left|X_{1}\right|=n / m$, we have $\left|A_{0}\right| /\left|A_{1}\right|=n / m$.

By Lemma 2] the induced subgraph $Y$ is semi-regular. Let $d=\delta(Y)=\delta_{A_{0}}$ and $F=N(A)$, we have $\delta_{A_{1}}=n d / m$. Since every vertex of $A_{0}$ has $m-d$ neighbours in $F$ and every vertex of $A_{1}$ has $n-n d / m$ neighbours in $F$, we have $|F|=\kappa(X) \geq m-d+n-n d / m=m+n-(m+n) d / m$. Вy $\kappa(X)<\delta(X)=m$, we have $d>m n /(m+n)$. Thus, $|A|=\left|A_{0}\right|+\left|A_{1}\right| \geq d+n d / m=(m+n) d / m>$ $n \geq m>\kappa(X)$. By Lemma 3. we deduce a contradiction.

The theorem then follows.

\section{Acknowledgements}

The author thanks the referees for many helpful suggestions and comments.

\section{References}

1. Hamidoune, Y.O.: Sur les atomes d'un graphe orienté. C.R.Acad. Sc. Paris Ser. A 284 (1977) 1253-1256

2. Liang, X., Meng, J.: Connectivity of Bi-Cayley Graphs. accepted by Ars. Comb.

3. Mader, W.: Über den Zusammenhang symmetricher Graphen. Arch. Math. 21 (1970) 331-336

4. Mader, W.: Minimale n-fach kantenzusammenhängende Graphen. Math. Ann. 191 (1971) 21-28

5. Mader, W.: Ein Eigenschaft der Atome endlicher Graphen. Arch. Math. 22 (1971) $331-336$

6. Meng, J.: Connectivity of vertex and edge transitive graphs. Discrete Appl. Math. 127 (2003) 601-613

7. Tindell, R.: Connectivity of Cayley digraphs. In: Du D.Z., Hsu D.F.(Eds.): Combinatorial network theory. Kluwer Academic Publishers, The Netherlands (1996) 41-64

8. Watkins, M.E.: Connectivity of transitive graphs. J.Comb. Theory 8 (1970) 23-29 